\title{
Educación para la salud y justicia social basada en el enfoque de las capacidades: Una oportunidad para el desarrollo de la salud pública
}

\author{
Education for health and social justice based on the focus on \\ capabilities: An opportunity for public health development
}

Lilliana Villa-Vélez (https://orcid.org/0000-0001-5571-4121) ${ }^{1}$

${ }^{1}$ Facultad Nacional de Salud Pública, Universidad de Antioquia. Cl. 67, 53108. Medellín Antioquia Colombia. liliana.villa @udea.edu.co.

\begin{abstract}
Theoretical research on health education is presented, which is proposed from an emancipatory perspective and constitutes an opportunity to promote health in communities because it makes it possible to explain a concept of social justice. The vision of social justice that is put forward in this article is focused on the capabilities that are understood as a set of real opportunities for people to achieve the life they aspire to. This approach is relevant for public health work because it is centered around human development, allows the subject to be incorporated into a dialectical relationship with society and focuses on real opportunities. The differences between public health approaches are considered to understand social justice and the relevance of a health concept that makes it possible to transcend the biomedical approach focused on disease. The importance of advancing towards a pedagogical development of health education that exceeds the perspective of education focused on behavioral changes is acknowledged, in order to understand it as a social and political process with an emancipatory humanist base as an opportunity for the construction of public health.
\end{abstract}

Key words Education, Health education, Social justice, Public health, Subject
Resumen Se presenta una investigación teórica sobre la educación para la salud, la cual se propone desde una perspectiva emancipadora y constituye una oportunidad para promover la salud en las comunidades porque permite explicitar una concepción de justicia social. La visión de justicia social que se plantea en este artículo está centrada el enfoque de las capacidades que se entienden como un conjunto de oportunidades reales para que las personas tengan la vida que valoran. Este enfoque es pertinente para el trabajo en salud pública porque está centrado en el desarrollo humano, permite incorporar al sujeto en relación dialéctica con la sociedad y se enfoca en las oportunidades reales. Se consideran las diferencias entre las corrientes de salud pública para entender la justicia social y la relevancia de una concepción de salud que permita trascender el abordaje biomédico centrado en la enfermedad. Se reconoce la importancia de avanzar hacia un desarrollo pedagógico de la educación para la salud que supere la perspectiva de educación centrada en los cambios de comportamiento, para entenderla como un proceso social y político con base humanista emancipadora como oportunidad para la construcción de la salud pública.

Palabras clave Educación, Educación en salud, Justicia social, Salud pública, Sujeto 


\section{Introducción}

En la comunidad académica no existe un consenso acerca de la concepción de justicia social que debe considerarse para el desarrollo de la salud pública y tampoco todas las corrientes de salud pública dejan explícita su idea de justicia. De igual manera desde la salud pública hay discrepancias sobre la forma como debería organizarse la sociedad para lograr mayor equidad en salud a partir de las diferentes corrientes de pensamiento. Este asunto resulta problemático frente a la toma de decisiones y requiere mayor profundidad en el análisis teórico dado que la justicia social es una cuestión inherente a la salud pública.

En lo que atañe a la Educación para la Salud (EpS) el panorama tampoco está claramente definido, no existe una sola concepción sobre la EpS y la falta de consenso implica un ejercicio académico que permita tomar posición como salubristas e investigadores a partir de la reflexión. Como punto de partida se asume que la EpS debe tener unas características que busquen la emancipación, es decir, que sea liberadora porque apunta a la transformación de la realidad, en el sentido que propone Freire': una "praxis que es la reflexión y acción de los hombres sobre el mundo para transformarlo"'(p.51). Se trata entonces de una EpS emancipadora que parte de una reflexión pedagógica humanista en la que rescata al sujeto en relación dialéctica con el mundo y con los otros, una liberación de los hombres en la que no son considerados meros "objetos" de la acción liberadora "sobre" y "para" quienes se realiza la acción, sino que se da "con" ellos'; es una forma de ganar conciencia de la realidad y luchar por una libertad que permita alcanzar la justicia social.

El objetivo de este artículo es mostrar que la EpS emancipadora constituye una oportunidad para construir la salud con las comunidades que viven injusticias sociales, porque permite promulgar una salud pública con una concepción de justicia social explícita que aquí se propone desde el enfoque de las capacidades. Para tal efecto se desarrolla el texto en dos secciones, en la primera se presentan las razones por las cuales el enfoque de justicia social basada en las capacidades es pertinente pensar la salud pública (en esta se incluyen asuntos en relación con los derechos humanos y las corrientes de salud pública) y en la segunda la necesidad de que la salud pública considere una EpS emancipadora que contribuya al fortalecimiento de capacidades para que las personas logren la vida que valoran.

\section{La justicia social basada en el enfoque de las capacidades como oportunidad para el trabajo teórico y práctico en salud pública}

Las injusticias sociales afectan profundamente las oportunidades de las personas para tener una vida buena, entendidas estas oportunidades como medio para fomentar el desarrollo huma$\mathrm{no}^{2}$. De la misma forma limitan las oportunidades para tener una buena salud -entendida como vida digna- ya que “...una de las libertades más importantes de las que podemos gozar es la de no estar expuestos a enfermedades y causas de mortalidad evitables"3. Para la salud pública se hace necesario considerar las injusticias sociales que limitan las oportunidades de las personas para tener una buena salud; se ha documentado que las pobres condiciones sociales desde la infancia llevan a tener una peor salud a lo largo de la vida $\mathrm{y}$ donde hay mayor inequidad hay peores condiciones de salud ${ }^{4}$. Esta es una cuestión central inclusive desde la visión causalista de la Comisión de Determinantes Sociales de la Salud, en la que llaman la atención sobre los debates alrededor de la equidad, insistiendo en que no solo se trata de buenos o malos resultados en salud, sino que es un asunto fundamentalmente de oportunidades ${ }^{4}$.

La equidad en una sociedad da cuenta de la forma como se organizan las instituciones y los grupos sociales, por lo tanto, es relevante desde el punto de vista moral que las acciones de la salud pública se piensen para generar oportunidades sociales, especialmente para quienes están en desventaja social; en este sentido la equidad tiene implícita una idea de justicia ${ }^{5}$.

Retomo dos perspectivas teóricas para abordar la justicia social, la liberal y la igualitarista social. La primera, propuesta por John Locke como "libertad contractual", parte del supuesto de que el contrato social se da entre hombre libres e iguales ${ }^{5}$, elemento también incorporado por el utilitarismo y la visión de la justicia distributiva.

Desde el utilitarismo se considera la justicia social en función de la búsqueda de los derechos humanos de libertad personal y propiedad privada, mercado y libre competencia, como valores que sustentan el orden capitalista; en esta perspectiva la utilidad significa el grado en que las leyes e instituciones jurídicas promueven la satisfacción del deseo de la mayor cantidad de individuos, al menor costo posible ${ }^{6}$. De acuerdo con Mill ${ }^{7}$ esta perspectiva propone el sacrificio de un individuo mediante la renuncia a su felicidad por el bienestar de la mayoría, aunque no propone que la sociedad sacrifique a los otros, sí lo 
hace en el ámbito del libre albedrio individual, en el que cada individuo decide por su propio bienestar y felicidad. Una de las críticas que se hace al utilitarismo de Mill' ${ }^{7}$ es que ubica al individuo como un medio y no como fin, lo cual puede ser cuestionado porque la pérdida de la libertad de unos no se compensa porque algunos gocen de mayor bienestar ${ }^{7}$, incluso si estos últimos son mayoría, esto entraría en contradicción con una salud pública que parte de una perspectiva de equidad. Esta perspectiva utilitarista ha permeado los sistemas de salud desde la lógica de la "visión de prioridad" para evaluar la calidad de los $\operatorname{mismos}^{5,7}$, dejando de lado la participación de las personas en la definición de sus necesidades.

La justicia distributiva tiene diferentes matices. Uno de sus principales representantes es Rawls, quien entiende la justicia social como principios normativos para asignar los derechos y deberes en las instituciones sociales y de esta manera determinar la correcta distribución de las cargas y beneficios de la vida social ${ }^{8}$; esta perspectiva presenta la justicia como equidad y se centra en asuntos formales para que todos tengan las mismas oportunidades. Este enfoque ha sido ampliamente criticado al interior del liberalismo porque se centra en la igualdad de bienes y no reconoce que su transformación en capacidades cambia de una persona a otra ${ }^{9}$, y por lo tanto se terminan maximizando los bienes a costa de algunas personas o grupos, porque el principio de la diferencia -que plantea que por mucho que se mejore la situación de una de las personas, no habrá ganancia a menos que la otra también se beneficie- ${ }^{8}$, no alcanza a proteger a todos en el ámbito de la influencia de las contingencias que se presentan en la sociedad, como las del mercado.

Otro de los matices desde una perspectiva liberal de justicia distributiva es el enfoque de las capacidades que parte de cuestionamientos a algunos enunciados de la teoría Rawlsiana ${ }^{10}$. Desde esta visión se propone que la justicia no puede ser indiferente a las vidas que las personas pueden realmente vivir y se hace énfasis en que: “(...) tenemos razón para estar interesados no solo en la clase de vidas que conseguimos vivir, sino también en la libertad que realmente tenemos para escoger entre diferentes estilos y modos de vida" ${ }^{\prime 10}$. Desde esta perspectiva, el foco de la justicia está centrado en la capacidad como libertad de elección de un conjunto de funcionamientos alternativos. Asimismo, plantea que las realizaciones sociales deben evaluarse desde el punto de vista de las capacidades que la gente tiene -entendidas como un conjunto de oportu- nidades reales ${ }^{10}$ - y concibe el desarrollo como un proceso de expansión de las libertades de las personas ${ }^{2}$, el cual se ve limitado por los problemas de la injusticia.

Un punto importante es que este enfoque está centrado en el desarrollo humano porque pone su atención en el sujeto, en la medida en que enfatiza que "los elementos más importantes de la calidad de vida de las personas son plurales y cualitativamente distintos" ${ }^{\prime 1}$. Para Sen se vincula estrechamente el concepto de capacidad al aspecto de oportunidad de la libertad, en la medida en que las oportunidades son "comprehensivas" -tienen en cuenta el proceso de elección- y no solo un asunto entendido como una "culminación" o un fin ${ }^{10}$. Este autor expone que las oportunidades sociales y los funcionamientos valiosos -entendidos como actividades o estados-, son la clave para el desarrollo; por ejemplo, argumenta cómo el mayor nivel educativo de la población en algunos países, contribuye a aprovechar las oportunidades y propone que el éxito del proceso impulsado por la política social permite mejorar la calidad de vida de las personas ${ }^{2}$.

De estos planteamientos se desprende que las oportunidades no pueden ser solo sociales, ya que dejarían por fuera las posibilidades propias de cada individuo; como respuesta, Nussbaum retomando los trabajos de Jonathan Wolff y Avner De-Shalit sobre la seguridad del futuro para el uso de las capacidades y la noción de funcionamiento fértil -que tiende a favorecer otras capacidades que se le relacionan-, propone el concepto de las capacidades combinadas -libertades sustanciales- que comprenden tanto las condiciones sociales, políticas y económicas, como las capacidades internas, estas últimas corresponden a "estados de la persona" que son cambiantes y pueden ser fortalecidos en las interacciones sociales ${ }^{11}$.

Aunque hay criticas al enfoque de las capacidades asociadas a su timidez para plantear la necesidad de hacer transformaciones estructurales al modelo capitalista ${ }^{12}$, se concibe que este enfoque tiene elementos valiosos para pensar el desarrollo de las capacidades en relación con una idea de justicia social, asuntos que se articulan con la EpS emancipadora en términos del lugar que da a la agencia del sujeto, porque lo que debe ser igual para todos es la posibilidad de elección y no la capacidad.

Por otra parte, la justicia social desde matices igualitaristas apunta a la garantía de las mismas oportunidades y condiciones para todos. Si bien Marx no plantea una teoría de justicia, puede 
inferirse en su postura una visión de las injusticias sociales desde la relación laboral, en la que la vida se vuelve un mero medio de supervivencia porque se enajena el producto de la actividad humana; explica como en las relaciones se produce el "trabajo enajenado" en el que se perpetúan las injusticias ${ }^{13}$.

Desde el igualitarismo se propone una perspectiva de justicia orientada desde la máxima: "De cada cual, según sus capacidades; a cada cual, según sus necesidades"14. Esta visión propone que hay que cambiar el modelo capitalista que es el que genera las injusticias, lo cual está en contraposición con las perspectivas liberales -incluida la de las capacidades-, porque cuestiona la idea de que los contratos se dan entre hombres libres e iguales, argumentando que en la realidad la desigualdad está dada en la forma de apropiación de los medios de producción ${ }^{15}$. Aunque existe divergencia entre estas posturas, podría pensarse que en ambas visiones se parte las diferencias entre los sujetos.

Cabe enfatizar que todas estas perspectivas de justicia social confluyen en la práctica de la salud pública en la actualidad y es importante reconocerlas y explicitarlas como se mencionará más adelante.

\section{Los derechos humanos, las capacidades y la Educación para la Salud}

El enfoque de las capacidades complementa el enfoque de los derechos humanos y ambos responden a una perspectiva liberal de justicia social ${ }^{16}$. Para Sen la capacidad se refiere a la oportunidad para ser y hacer lo que se valora y los derechos humanos constituyen una de esas oportunidades, son una vía política para la reivindicación ética de la libertad ${ }^{10}$; pero la capacidad no queda reducida a un derecho -entendido como recurso o "bien elemental"-, sino que se enfoca en la libertad real para conseguir combinaciones alternativas de funcionamientos ${ }^{17}$. Por el contrario, Nussbaum propone unas capacidades centrales que entiende como derechos humanos que un orden político estaría obligado a procurar, no solo en un sentido instrumental, sino que tienen un valor en sí mismas para una vida completamente humana ${ }^{11}$.

La salud entendida como vida está relacionada con esas capacidades; en este sentido Venkatapuram ${ }^{18}$ subraya que la salud de una persona está en función de sus habilidades para ser y hacer algunas cosas básicas, cada ser humano tiene derecho a tener la capacidad de ser saludable, la salud sería entonces para él una metacapacidad entendida como capacidad global para lograr un conjunto de capacidades básicas para ser y hacer cosas que conforman una vida buena ${ }^{18}$.

Entender estas capacidades fundamentales como derechos aporta a la salud pública porque permite concretar esta visión de justicia social. El uso del lenguaje de derechos en el enfoque de las capacidades está justificado porque: prioriza asuntos urgentes sobre los que hay que actuar; le da responsabilidad al Estado para garantizarlos; favorece la posibilidad de elección y autonomía; y corresponden a asuntos sobre los que hay algunos acuerdos (aunque el debate continúa vigente) $)^{13}$.

Por otra parte, la salud y la educación son derechos interdependientes, ambos hacen parte del derecho a una vida digna -la salud es entendida aquí como metacapacidad-. Para $\operatorname{Sen}^{10}$ la buena salud está en relación con la educación en gene$\mathrm{ral}^{10}$; así, unas pobres condiciones de salud menoscaban la posibilidad de la educación y a la vez se requieren procesos educativos para mejorar la salud, porque la comprensión sobre la propia salud puede estar limitada por la falta de conocimientos médicos ${ }^{10}$.

Desde esta perspectiva de justicia se podría reconocer la EpS como derecho y necesidad de las personas, en la medida en que aporta a la garantía de la salud como un funcionamiento básico para la vida buena; pero esencialmente como una capacidad en términos de oportunidad para decidir sobre la propia salud. Serían entonces el derecho a la salud y el derecho a la EpS derechos interdependientes; de forma que la EpS emancipadora debería hacer parte de las políticas públicas de los países como apuesta pedagógica para hacer frente a las injusticias sociales y el enfoque de las capacidades constituye un marco teórico valioso para el desarrollo de esas políticas ${ }^{16}$.

Por otra parte, la EpS por sí sola no es suficiente para el logro de las capacidades para vivir la vida que se valora. Es importante reconocer que la educación -en general- no resuelve todo, ni genera las transformaciones sociales, pero en articulación con otras dimensiones aporta al cambio en los sujetos y de esta manera a la reestructuración de los poderes en las sociedades. La educación es un elemento central del enfoque de las capacidades porque:

(...) forma las aptitudes ya existentes en las personas y las transforma en capacidades internas desarrolladas de muchas clases. Esta formación es valiosa en sí misma, pero también es una fuente de satisfacción para la vida. Ejerce asimismo una 
función capital para el desarrollo y la ejercitación de otras muchas capacidades humanas: es, pues, un «funcionamiento fértil» de suma importancia para abordar los problemas de la desventaja y de la desigualdad $^{11}$ (p.266).

Dado que la salud pública está atravesada por injusticias sociales, es entonces la EpS emancipadora una alternativa para rescatar las potencialidades del sujeto, que a través de la comprensión de su realidad se va transformando desde la reflexión-acción ${ }^{1-19}$. Es decir, la EpS posibilita la emergencia del sujeto como ser político en la vía de las capacidades combinadas porque, la educación es fuente de opciones laborales y de poder político ${ }^{11}$ y constituye un camino para el reconocimiento de la salud que las personas valoran.

\section{Corrientes de salud pública para enmarcar la justicia social}

En la salud pública se requiere pensar una justicia social en la cual se pueda avanzar hacia la transformación de la realidad de manera reflexionada. No se trata de cuestionar si incorporamos o no la justicia social en el abordaje de la salud pública, sino cuál es pertinente, porque como plantea Venkatapuram ${ }^{18}$ las teorías de la justicia no hacen explícito el valor de la salud y esto puede llevar a concepciones de justicia social que produzcan más injusticias ${ }^{18}$.

Si bien en salud pública hay múltiples corrientes, pueden identificarse dos tendencias principales en las que el salubrista como sujeto moral puede situarse ${ }^{20}$. Por una parte, está la perspectiva tradicional de origen anglosajón que se enfoca en la enfermedad y construye su conocimiento desde la epidemiologia clásicaa ${ }^{21-23}$; por tanto, tiende a una explicación reduccionista y fragmentaria de la realidad, prioriza la lógica de asociación causa-efecto, reduce las nociones de exposición/vulnerabilidad a problemas individuales probabilísticos y se centra en los factores de riesgo para actuar ${ }^{24}$.

Por otra parte tenemos una perspectiva crítica con múltiples matices y tensiones; entre ellas, la teoría eco social que cuestiona la dicotomía entre el mundo biológico y social ${ }^{25}$; la escuela crítica europea de Pompeu Fabra que enfatiza las categorías salud y enfermedad como categorías políticas ${ }^{26}$; y la Medicina Social Latinoamericana y Salud Colectiva que ha sido permeada por movimientos académicos y políticos críticos, dando relevancia al ejercicio de la salud pública desde el bienestar, la justicia social y el sujeto como productor de salud. Esta última comprende una visión ética coherente con la propuesta de EpS emancipadora.

La salud pública tradicional responde a una concepción de justicia liberal utilitarista en la medida en que es individualista, prioriza la propiedad privada y la distribución de recursos públicos, es individual y focalizada ${ }^{14}$; no cuestiona la estratificación social (que es inherente a los sistemas sociales); tiene una visión causal y determinista que es limitada para dar cuenta de la libertad y el florecimiento humano ${ }^{27}$. Dentro de la perspectiva crítica la de la Medicina Social Latinoamericana y Salud Colectiva se presenta una posición de justicia igualitarista social que parte de entender la inequidad como producto de las relaciones de poder que generan las desigualdades $^{14}$.

Desde el enfoque de las capacidades se da relevancia al derecho a la dignidad, esto se sustenta en una perspectiva kantiana que toma al ser humano como un fin en sí mismo y no solo como un medio; por tanto, aunque no propone un cambio radical al modelo capitalista, tiene intrínseco un interés por un relacionamiento social más equitativo. En esta medida pueden pensarse un elemento común desde el punto de vista ético, entre la corriente liberal redistributiva y la igualitarista de la perspectiva latinoamericana, teniendo en cuenta que para pensar la justicia social se reconoce al sujeto como fin: "(...) Un diálogo entre la idea del 'sujeto moral' y el 'sujeto histórico-político"'14.

\section{La educación para la salud y justicia social desde el enfoque de las capacidades}

Pueden reconocerse dos tendencias en EpS, una tradicional que corresponde a los modelos informativo y persuasivo-motivacional enfocados en los cambios de comportamiento y otra crítica $^{28,29}$. La tradicional tiene en su base una concepción pedagógica bancaria que da cuenta de una educación en la que hay una relación no dialógica entre el educador y el educando, es el educador quien educa, sabe, piensa, habla, disciplina, prescribe, actúa, escoge el contenido programático, usa la autoridad del saber sobre la libertad del educando y se constituye el sujeto del proceso educativo, mientras los educandos son objetos pasivos y acríticos en su aprendizaje ${ }^{1}$; por el contrario, la educación crítica da pie a pensar una educación emancipadora. El ejercicio de la EpS reflexionada conduce a una decisión entre ambos tipos de educación, con base en las experiencias personales del educador, pero también 
en las imposiciones del medio institucional y la inercia que genera la educación bancaria.

Así como el insuficiente desarrollo teórico de la justicia social en la salud pública puede conducir la toma de decisiones que perpetúan las injusticias sociales ${ }^{18}$, la EpS tradicional puede validar los mismos procesos de injusticia dado que se da de manera impositiva y desconoce al sujeto y sus condiciones.

La Educación Popular, como pedagogía crítica, va en contravía a la educación bancaria y permite pensar una EpS con un fundamento político intencionado que busca contribuir a la construcción de una nueva sociedad más justa y democrática $^{30}$. Por esta vía puede pensarse la EpS en los espacios comunitarios y clínicos como un escenario que aporte a la transformación del sujeto político, permitiéndole reflexionar sobre su realidad y actuar en coherencia; asimismo, la EpS vista desde este enfoque, se entiende como capacidad combinada y no estaría limitada al desarrollo de habilidades individuales.

Tradicionalmente se ha entendido la EpS como: (...) oportunidades conscientemente construidas para el aprendizaje que involucran algún tipo de comunicación diseñada para mejorar la alfabetización en salud, incluyendo la mejora del conocimiento y el desarrollo de habilidades para la vida que son conducentes a la salud individual y comunitaria $^{31}$; en este sentido, la EpS no está aislada de la educación en general porque la alfabetización en salud depende en gran medida de los niveles de alfabetización en general.

La noción de alfabetización en salud es muy limitada y apunta hacia un enfoque de la justicia social utilitarista en la medida en que parte de una visión de la salud centrada en la enfermedad y pretende una educación instrumental que considera al individuo como medio para el logro de indicadores en salud, desconociendo sus necesidades e intereses. Aunque el enfoque de las capacidades permite pensar al sujeto como fin en el proceso educativo, también tiende a una visión de salud centrada en la enfermedad -desde Sen-; esta es una limitación que podría superarse pensando la capacidad de tener una vida buena en articulación con otras capacidades, como metacapacidad, en la vía que plantea Nordenfelt, citado por Venkatapuram: "la salud como capacidad de lograr un conjunto de objetivos vitales"18.

En este orden de ideas, la EpS emancipadora requiere integrar concepciones más amplias de la salud y la educación para poder avanzar coherentemente, se trata de prácticas pedagógicas que sean dialógicas y participativas, que reconozcan la pluralidad y que contribuyan a las transformaciones individuales y sociales. Como punto de partida, la EpS puede entenderse como: "Un conjunto de prácticas pedagógicas de carácter participativo y emancipador que abarca varios campos de acción y tiene como objetivo movilizar y sensibilizar a las personas y hacerlas conscientes frente a situaciones individuales y colectivas que interfieren en la calidad de vida" 32 .

A pesar de que la EpS se ha presentado como un asunto importante en la salud pública, en un marco con orientación predominantemente biomédica tiende a asumirse instrumental, y se olvida que su papel y desarrollo dependen de la corriente de la salud pública en la cual se inscribe $^{33}$; esto tiene implicaciones para la EpS porque deja de lado que la salud pública tiene implícita una tarea educativa con sus correspondientes compromisos éticos y políticos que le exigen una permanente reflexión pedagógica para no reproducir las injusticias sociales.

En la salud pública no puede desconocerse la importancia que tiene la educación para los procesos de socialización, entendidos como “(...) la inducción amplia y coherente de un individuo en el mundo objetivo de una sociedad o en un sector de él"34. Dado que la socialización primaria que se da en la familia desde la niñez puede estar cargada de injusticias, la socialización secundaria desarrollada en el ámbito institucional constituye una oportunidad para "redistribuir las cargas sociales" -y cambiar las condiciones para la socialización primaria en las generaciones venideras, porque está en función de la división del trabajo y la distribución del conocimiento en la sociedad. En este sentido, superar el abordaje tradicional de la EpS implica comprender su papel frente a los procesos de reproducción de las injusticias.

Si partimos de la idea de que toda práctica pedagógica es moral, la EpS emancipadora no se ocuparía sólo de asuntos informativos en relación con la salud (contenidos instrumentales), sino de una comprensión de las relaciones con los otros y de las reflexiones éticas para comprender el sentido de la acción moral desde una perspectiva de justicia social explícita. El desarrollo de la EpS emancipadora desde el enfoque de las capacidades aporta a una salud pública diferente porque contribuye a la construcción de la vida buena de carácter pluralista ${ }^{35}$, en la medida en que se centra en favorecer el proceso de elección de alternativas de la persona, “(...) en particular las otras alternativas que ella podría elegir dentro de su habilidad real para hacerlo" ${ }^{10}$. En este orden de ideas, la posibilidad de elección está dada en 
la relación dialéctica sujeto-sociedad como se ha planteado.

Por esto se requiere una reflexión pedagógica alrededor de la educación que oriente la práctica; es decir, para poder actuar tenemos que comprender; se necesita construir más teoría en la educación para que ésta sea realmente crítica, porque a veces la práctica se convierte en acciones desarticuladas y se pierde de vista que la educación emancipadora lucha por la libertad para construir la justicia social ${ }^{32}$. En este sentido Freire plantea que "la educación verdadera es praxis, reflexión y acción del hombre sobre el mundo para transformarlo"19.

\section{A manera de conclusión}

No solo es importante reconocer la salud que las personas valoran sino también la oportunidad que tienen para construir esa salud. Esto con frecuencia se olvida en los sistemas de salud que operan con una lógica de la utilidad o basada en los recursos y no consideran lo que las personas valoran desde sus subjetividades. En contraste, el enfoque de las capacidades que se viene mencionado ofrece una posibilidad de pensar la equidad ya que reconoce al sujeto situado y sus oportunidades reales: “(...) la ventaja de una persona se juzga menor que la de otra si tiene menos capacidad -menos oportunidad real- de lograr esas cosas que tiene razón para valorar" ${ }^{\prime 10}$.
Pensar el sujeto social desde la EpS permite varios encuentros: 1) con el enfoque de las capacidades porque pensar la educación implica considerar las oportunidades sociales y las habilidades personales -la EpS como capacidades combinada-; 2) con la Medicina Social Latinoamericana y Salud Colectiva porque la EpS emancipadora contribuye a mejorar la salud en las poblaciones en la vía del desarrollo del sujeto-sociedad en relación dialéctica, en este sentido la sociedad no es una abstracción frente al individuo: “(...) el individuo es el ser social"12; 3) entre el enfoque de las capacidades y las perspectivas latinoamericanas de salud pública que reconocen al sujeto para pensar la justicia social.

Finalmente, esta propuesta parte de pensar en la salud pública las posibilidades reales que tienen las personas para la salud-vida que valoran desde los cuestionamientos centrales del enfoque de las capacidades: “¿qué son realmente capaces de hacer y de ser las personas? y ¿qué oportunidades tienen verdaderamente a su disposición para hacer o ser lo que puedan?"11. Las respuestas a las preguntas pueden ser construidas desde la EpS entendida como un proceso social y político. Se propone una educación con base humanista liberadora, que movilice a la concienciación, reconfigure la relación entre los actores del proceso educativo y esté basada en el diálogo y la participación; a la vez que se articule con una salud pública que explicite su apuesta por la justicia social.

\section{Financiación}

Esta investigación teórica fue desarrollada en el marco de la formación doctoral en salud pública, para la cual se ha contado con financiación de COLCIENCIAS - doctorados nacionales. 


\section{Referencias}

1. Freire P. Pedagogía del oprimido. $2^{\text {a }}$ ed. Uruguay: Siglo XXI; 2005.

2. Sen A. Los fines y los medios del desarrollo. En: Desarrollo y libertad. Bogotá: Editorial Planeta; 2000. p. 54-75.

3. Sen A. La salud en el desarrollo. En: Discurso inaugural pronunciado en la 52a Asamblea Mundial de la Salud: Boletín Organización Mundial de la Salud. Ginebra: OMS; 2000. p. 16-21.

4. Solar O, Irwin A. Towards a Conceptual Framework for Analysis and Action on the Social Determinants of Health: In: Discussion paper for the Commission on Social Determinants of Health. Genebra: CSDH/WHO; 2005. p. 1-33.

5. Hernández-Alvarez M. El Concepto de Equidad y el Debate sobre lo Justo en Salud. Rev Salud Pública Univ Nac Colomb 2008;10(1):72-82.

6. Bentham J. Introducción a los principios de moral y legislación. Buenos Aires: Claridad; 2008.

7. Mill J. El utilitarismo. En: ¿Qué es el utilitarismo?. Madrid: Alianza Editorial; 1984. p. 44-75.

8. Rawls J. Teoría de la justicia. México D.F.: Fondo de Cultura Económica; 2010.

9. Sen A. ¿Igualdad de qué? En: Conferencia sobre los valores humanos pronunciada en la Universidad de Stanford. California: Universidad de Stanford; 1979. p. 133-156.

10. Sen A. La idea de la justicia. Buenos Aires: Taurus; 2009.

11. Nussbaum M. Crear capacidades. Propuesta para el desarrollo humano. Barcelona: Paidos; 2012.

12. Campana M. Para una lectura crítica del desarrollo humano. Andes 2013; 24(2):00-00.

13. Marx K. Manuscritos de economía y filosofía. Madrid: Alianza Editorial; 2001.

14. Marx K. Crítica del programa de Gotha. Moscú: Biblioteca Virtual; 1977.

15. Hernández-Alvarez M. Desigualdad, inequidad e injusticia en el debate actual de la salud: posiciones e implicaciones. En: Taller Latinoamericano de Determinantes Sociales de la Salud. Ponencia presentada a la mesa "Problemas éticos: desigualdad, inequidad e injusticia". México: Asociación Latinoamericana de Medicina Social (ALAMES); 2008. p. 1-16.

16. Nussbaum M. Capabilities and Human Rights. Fordham Law Rev 1997; 66(2):273-300.

17. Sen A. Nuevo examen de la desigualdad. Madrid: Alianza Editorial; 1992.

18. Venkatapuram S. Health Justice. An Argumento from the Capabilities Approach. Cambridge: Polity Press; 2011.

19. Freire P. La educación como práctica de la libertad. $45^{\mathrm{a}}$ ed. Uruguay: Siglo XXI; 1997.

20. Peñaranda F. Salud Pública, justicia social e investigación cualitativa: hacia una investigación por principios. Rev Fac Nac Salud pública 2015; 33(1):103-111.

21. Granda E. La salud y la vida. Vol 2. Quito: Ministerio de Salud Pública de Ecuador, OPS/OMS; 2009.
22. Granda E. El saber en salud pública en un ámbito de pérdida del antropocentrismo y ante una visión de equilibrio ecológico. Rev Fac Nac Salud Pública 2008; 26:65-90.

23. Granda E. La salud y la vida. Vol 1. Quito: Ministerio de Salud Pública de Ecuador, OPS/OMS; 2009.

24. Breilh J. La determinación social de la salud como herramienta de transformación hacia una nueva salud pública (salud colectiva). Rev Fac Nac Salud pública 2013; 31(1):13-27.

25. Krieger N. Proximal, distal, and the politics of causation: What's level got to do with it? Am J Public Health 2008; 98(2):221-230.

26. Navarro V. Concepto actual de salud pública. Salud Pública 1997; 49-54.

27. Peñaranda F. Sujeto, justicia social y salud pública. Cien Saude Colet 2015; 20(4):987-996.

28. Diaz-valencia PA. Concepciones teóricas sobre la teoría en educación para la salud. Revisión sistemática. Invest Educ Enferm 2012; 30(3):378-389.

29. Serrano M. Líneas básicas en el estudio de la educación para la salud. En: Díaz de Santos, editor. Educación para la Salud. Madrid; 1990. p. 26-41.

30. Torres A. Educación Popular. Trayectoria y actualidad. Caracas: Universidad Bolivariana de Venezuela; 2011.

31. Nutbeam D. Health literacy as a public health goal: a challenge for contemporary health education and communication strategies into the 21st century. Health Promot Int 2000; 15(3):259-267.

32. Brasil. Ministério da Saúde (MS). Painel de indicadores do SUS No 6 Promoção da Saúde. Brasilia: OPAS/ OMS; 2009.

33. Peñaranda CF, Giraldo GL, Barrera PLH. La enseñanza de la educación para la salud: ¿una confrontación a la teoría y la práctica de la salud pública como disciplina? Rev Fac Nac Salud Publica 2015; 33(3):353-360.

34. Berger PL, Luckmann T. La construcción social de la realidad. Buenos Aires: Talleres Gráficos Color Efe; 1968.

35. Restrepo-Ochoa DA. La salud y la vida buena: aportes del enfoque de las capacidades de Amartya Sen para el razonamiento ético en salud pública. Cad Saude $\mathrm{Pu}$ blica 2013; 29(12):2371-2382.

Artículo presentado en 22/11/2017

Aprobado en 27/08/2018

Versión final presentada en 29/08/2018 